\section{Spectral Characteristics of Citrus Black Spot Disease}

\author{
Alireza Pourreza ${ }^{1}$, Won Suk Lee ${ }^{1,4}$, Mark A. Ritenour ${ }^{2}$, \\ and Pamela Roberts ${ }^{3}$
}

Additional INDEX wORDs. CBS, fungus, spectrometry, diagnosis, machine learning, band selection, classification

\begin{abstract}
Summary. Citrus black spot (CBS) is a fungal disease caused by Phyllosticta citricarpa (synonym Guignardia citricarpa). CBS causes fruit lesions and significant yield loss in all citrus ( Citrus) species. The most distinguishing CBS symptom is called hard spot, which is a circular lesion with gray tissue at the center surrounded by a black margin. The spectral characteristic of CBS lesions was investigated and compared with the spectral signature of healthy fruit tissue to determine the best distinguishing wave band. Healthy and CBS-affected samples presented similar reflectance below $500 \mathrm{~nm}$ and above $900 \mathrm{~nm}$. However, healthy samples reflected more light between 500 and $900 \mathrm{~nm}$, especially within the visible band. Also, spectral reflectance of the same symptomatic lesion was acquired six times over a 2-month period to determine the variation of symptom's spectral signatures over time after being harvested. A two-sample $t$ test was employed to compare each pair of consecutive repetitions. The results showed that the spectral signature of the CBS lesion did not change significantly over 2 months. The wavelengths between 587 and $589 \mathrm{~nm}$ were identified as the distinguishing band to develop a monochrome vision-based sensor for CBS diagnosis. A support vector machine (SVM) classifier was trained using the spectral reflectance data at the selected bands to identify CBSaffected samples in each repetition. The overall CBS detection accuracies varied between $93.3 \%$ and $94.6 \%$.
\end{abstract}

$\mathrm{F}$ lorida citrus industry has been threatened by several, previously exotic citrus diseases during the past few years. Citrus canker (caused by Xanthomonas axonopodis pv. citri), a bacterial disease, was discovered in 1995 near the Miami International Airport (Gottwald et al., 2002) and despite all the efforts to eradicate the disease, it became widespread in Florida mainly because of the 2004-05 hurricanes (Stover et al., 2014). In 2005, another destructive bacterial disease called Huanglongbing (HLB) or citrus greening caused by Candidatus Liberibacter asiaticus and vectored by the asian citrus psyllid (Diaphorina citri) was discovered in Florida City, south of Miami (Gottwald et al.,

We would like to thank the Florida Specialty Crop Block Grant Program for funding this research. We would also like to express our sincere appreciation to Ce Yang, Chuanqi Xie, Daeun Choi, and Chuanyuan Zhao for their assistance in this study.

${ }^{1}$ Department of Agricultural and Biological Engineering, University of Florida, Gainesville, FL 32611

${ }^{2}$ Indian River Research and Education Center, University of Florida, Fort Pierce, FL 34945

${ }^{3}$ Southwest Florida Research \& Education Center, University of Florida, Immokalee, FL 34142

${ }^{4}$ Corresponding author. E-mail: wslee@ufl.edu.
2007). Again after a few years, HLB disease was found in all citrus producing counties in Florida and made a huge impact on the $\$ 9$ billion citrus industry in Florida (Pourreza et al., 2014). In Mar. 2010 , CBS was diagnosed on fruit in some groves near Immokalee, FL (Schubert et al., 2013). CBS is a fungal disease caused by $P$. citricarpa (Er et al., 2013). The disease was found in Taiwan, South Africa, and China in 1919, 1920, and 1936, respectively (Kotzé, 1981; Wang et al., 2012). Later in the 1980 s, CBS was officially found in coastal humid regions of Australia and caused huge yield losses for several years (Kotzé, 1981).

CBS causes fruit lesions and substantial yield loss in all citrus species. Sweet orange (Citrus sinensis) varieties such as the 'Valencia' are extremely susceptible to this disease. CBS causes a wide range of symptoms, but the most distinguishing one is called hard spot (Fig. 1), which is a circular lesion (with 3$10 \mathrm{~mm}$ diameter) with gray necrotic fatal tissue at the center embraced by a black margin. Hard spot becomes apparent at the time of fruit coloring just before harvesting (Dewdney et al., 2014). Fruit that have CBS lesions are nonvaluable for the fresh markets. Premature fruit drop is also a consequence of CBS disease in severe conditions. There is a long latent stage in the disease cycle in which the CBS symptoms are not apparent for several months after infection. Symptoms usually appear at the time of fruit ripening (Dewdney et al., 2014).

To reduce the spread of the disease, CBS must be efficiently controlled in the grove. In addition, manual detection of CBS symptoms at the packaging process is extremely difficult because they may be confused with blemishes caused by other disorders and the process is time consuming. Therefore, a rapid and accurate CBS identification technique can expedite the quality control process and help growers for better disease management. Computer vision-based sensors have been widely used for plant disease identification (Pourreza et al., 2015; Qin et al., 2012; Sankaran et al., 2010). However, using vision sensors for CBS identification has not been thoroughly investigated. Bulanon et al. (2013) analyzed hyperspectral images of CBS symptoms in the range of 480-950 $\mathrm{nm}$ (spectral resolution of $2.8 \mathrm{~nm}$ ) with the objective of determining the potential bands to develop a multispectral imaging sensor. They defined four wavelengths including $781,713,629$, and $493 \mathrm{~nm}$ as the selected bands for a multispectral image acquisition system. They achieved the overall accuracy of $96 \%$ using the four selected wavelengths and normalized difference vegetation index band ratio of $781 \mathrm{~nm}$ [near-infrared (NIR)] and $713 \mathrm{~nm}$ (red) as the input features.

\begin{tabular}{llll}
\hline $\begin{array}{l}\text { Units } \\
\begin{array}{l}\text { To convert U.S. to SI, } \\
\text { multiply by }\end{array}\end{array}$ & U.S. unit & SI unit & $\begin{array}{l}\text { To convert SI to U.S., } \\
\text { multiply by }\end{array}$ \\
\hline 25.4 & inch(es) & $\mathrm{mm}$ & 0.0394 \\
1 & micron $(\mathrm{s})$ & ${ }^{\mu \mathrm{m}}$ & $\begin{array}{l}1 \\
\left({ }^{\circ} \mathrm{C}-32\right) \div 1.8\end{array}$ \\
${ }^{\circ} \mathrm{F}$ & $\left.{ }^{\circ} \mathrm{C} \times 1.8\right)+32$
\end{tabular}




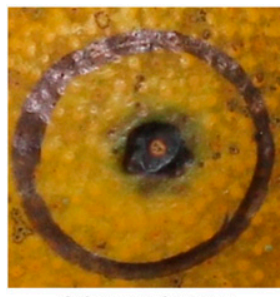

Advanced stage

(CBS-positive)

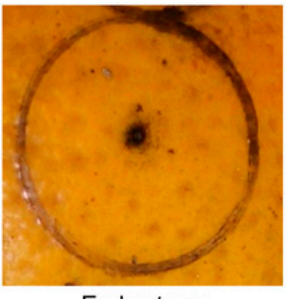

Early stage

(CBS-positive)

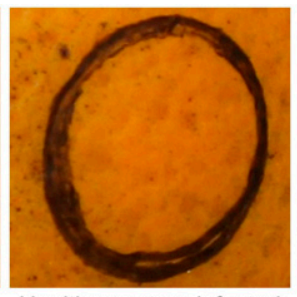

Healthy spot on infected sample (CBS-positive)

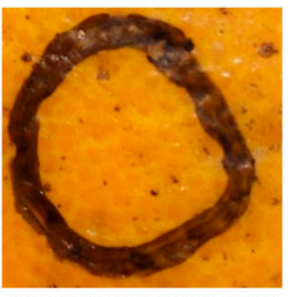

Healthy spot on healthy sample (CBS-negative)

Fig. 1. Images of two citrus black spot lesions (CBS positive) and two CBSnegative spots on peels of 'Valencia' sweet orange fruit.

The main goal of this research was to investigate the spectral signatures of CBS hard spot lesions using high spectral resolution data and determine the best wavelength for CBS identification. The specific objectives were to: 1) investigate the progress of the lesion development on fruit over time after harvest, 2) select the important wave bands for designing a customized and image acquisition system to CBS symptoms, and 3) evaluate the classification accuracy using the selected band as the input feature.

\section{Materials and methods}

Data collection. Healthy and CBS symptomatic citrus fruit of 'Valencia' sweet orange were collected from a citrus grove near Immokalee, FL, in Apr. 2014. To conduct the spectral measurement, the citrus samples were transferred to the postharvest laboratory in the Indian River Research and Education Center, University of Florida, Fort Pierce. A total of 134 citrus fruit including 104 CBS-positive and $30 \mathrm{CBS}$-negative samples were selected randomly for spectral measurement. Two or three CBS symptomatic hard spot lesions were selected and marked randomly on each of the CBS-positive samples to create the CBS-positive class. Also, two CBS asymptomatic spots on each of the CBS-positive samples and two or three spots on CBS-negative fruit were selected to create the CBSnegative class.

SPECTRAL REFLECTANCE MEASUREMENT. A portable spectrometer (USB2000+; Ocean Optics, Dunedin, FL) with spectral resolution range of $\approx 0.28$ to $0.38 \mathrm{~nm}$ and an optical resolution of $0.1 \mathrm{~nm}$ was used to measure the spectral reflectance of the target spots on the citrus fruit. A fiber optic reflection probe
(QR600-7-SR-125F; Ocean Optics) with a core diameter of $600 \mu \mathrm{m}$ was used to measure the spectral reflectance of the target spots. A reflection probe holder (RPH-1; Ocean Optics) was used to keep the same probe positioning to all the target spots at $45^{\circ}$ in all measurements. The light source (LS-1 Tungsten Halogen Light Source; Ocean Optics) of the spectrometer was turned on $45 \mathrm{~min}$ before measurement to ensure it reached its stable status. The optical reference standard was collected before each measurement using a certified $99 \%$ white reflectance standard (SRS-99-020; Labsphere, North Sutton, $\mathrm{NH}$ ).

The measurement was repeated six times on 11 Apr., 18 Apr., 2 May, 15 May, 29 May, and 13 June 2014. The CBS-negative samples were added to the dataset from the second repetition (18 Apr.). At the forth repetition of spectral measurement, a few samples began to decay. These decayed samples were removed before the fifth and sixth repetitions of spectral measurement. Color images of the citrus samples and the selected spots were taken as a reference of the sample status at the time of the spectral measurement. The citrus samples were kept at air temperature of $1{ }^{\circ} \mathrm{C}$ and humidity of $70 \%$ during the experiment period. The spectral range of the spectrometer was between 340 and $1030 \mathrm{~nm}$; however, the measured reflectance below $400 \mathrm{~nm}$ was very noisy. Therefore, the analysis was performed within the entire visible and part of NIR bands. In total, 1914 wavelengths between 400 and 1030 $\mathrm{nm}$ were available for the analysis process.

C OM PARISON B E T W E E N Repetitions. Two-sample $t$ test (Snedecor and Cochran 1989) was used to determine whether there is any significant difference between the spectral signatures of different repetitions. The averages of spectral reflectance and their first derivatives were compared between every two consecutive repetitions and also between the first and last repetitions for each class. All data analyses were conducted using MATLAB (version R20lla; MathWorks, Natick, MA).

Band SElection. To determine the most important wavelengths in CBS diagnosis, five feature ranking methods including $t$ test, KullbackLeibler distance, Chernoff bound, receiver operating characteristic, and Wilcoxon tests were employed (Liu and Motoda, 1998; Theodoridis and Koutroumbas, 2009). The results of each method included a vector of coefficients corresponding to the features (wavelengths). These coefficients represent the level of relevance of each wavelength for the classification process. The coefficient ranges for each method varied since different factors were considered for different feature ranking methods. Therefore, the $\mathrm{co}^{-}$ efficients in each method were normalized by the maximum obtained coefficient that belonged to the top wavelength ranked with the corresponding method. To select the most relevant bands, a combination of the entire feature ranking results was used to appoint the best set of nominated wavelengths, which were voted by all methods. For this purpose, all the wavelengths that had the coefficients greater than 0.99 in all methods were selected.

Classification. The selected bands were used to train a SVM binary classifier with two classes of CBS positive and CBS negative (Bishop, 2006). Classification was conducted for every repetition as well as the entire dataset. A 10-fold cross validation method was used in the classification process in which the dataset was randomly divided into 10 folds; 9 folds were used for training while the other fold was used for validation. The classification was repeated 10 times until all the samples were included at least once in the validation set.

\section{Results}

Dataset preparation. In total, 3102 spectral reflectances were measured during the six repetitions of the experiment. Two CBS-positive and 
two CBS-negative spots were shown on the citrus samples (Fig. 1). The dataset included spectral data of 1553 CBS positive ( 458 advanced stage and 1095 early stage) and 1460 CBS negative (1026 healthy spot on CBS-positive fruit and 434 healthy spot on CBS-negative fruit). Early and advanced stages of CBS lesions were confirmed by several observations conducted by plant pathologists. Early stage of a CBS hard spot lesion is a small and round spot in dark gray color, whereas the advanced stage of lesion shows a tan center surrounded by a brick-red border.
The average spectral reflectance of two CBS lesions (advanced and early stages) and two healthy (healthy spot on CBS-positive fruit and healthy spot on CBS-negative fruit) classes were shown (Fig. 2). The variation (SD) within each class and at each wavelength was illustrated (Fig. 3). The CBS-positive classes had less reflectance than healthy classes at the range of 500 to $820 \mathrm{~nm}$, which is more distinctive in the spectral reflectance curve of the advanced stage of the CBS-positive class. However, the CBS-negative spots reflected more at the visible band. In addition,

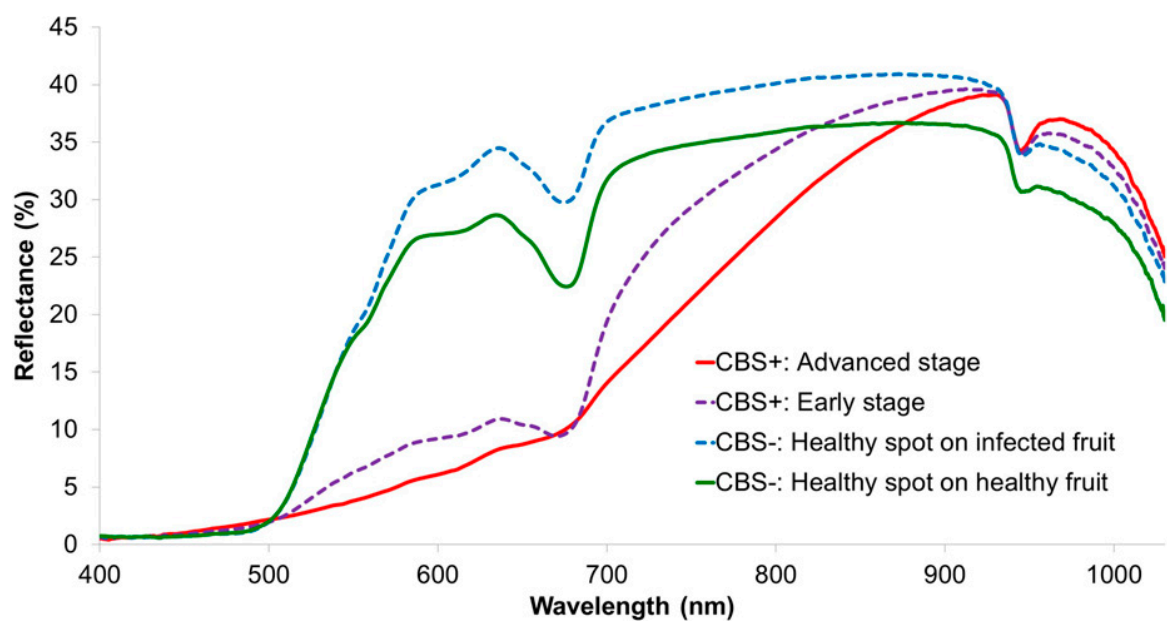

Fig. 2. Average spectral reflectance of citrus black spot (CBS) lesions in two classes (advanced and early stages of hard spot) and healthy spots in two CBS-negative classes (healthy spot on CBS-positive fruit and healthy spot on CBS-negative fruit) on peels of 'Valencia' sweet orange fruit within the visible and near-infrared spectral ranges.

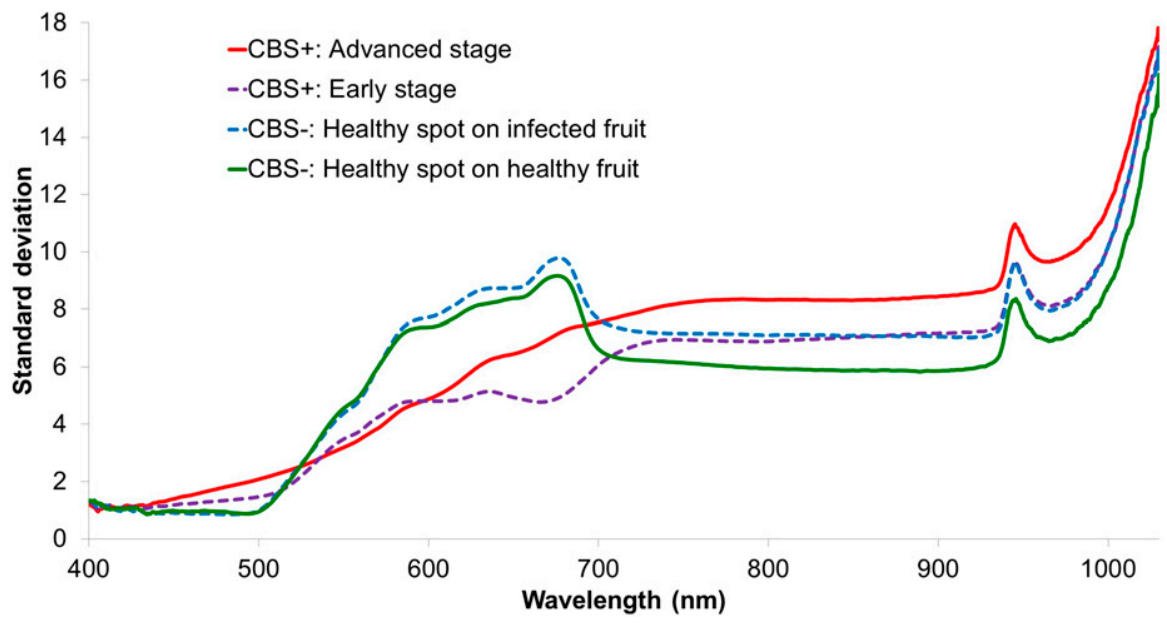

Fig. 3. Average standard deviations of spectral reflectance of samples in two citrus black spot positive (CBS-positive) classes (advanced and early stages of hard spot) and two CBS-negative classes (healthy spot on CBS-positive fruit and healthy spot on CBS-negative fruit) on peels of 'Valencia' sweet orange fruit within the visible and near-infrared spectral ranges.

both CBS-negative classes created very similar spectral signatures. It can be concluded that the nonsymptomatic parts on CBS-infected fruit had similar spectral characteristics to the healthy fruit. All classes had similar reflectance at the wavelengths below $500 \mathrm{~nm}$ with small standard deviations. The spectral signatures of the four classes at the wavelengths over $930 \mathrm{~nm}$ also generated very similar patterns. Since the spectrometer accuracy at the lower and upper bands of the detector range was not as accurate as the middle band, and the four classes generated similar spectral reflectance at the two ends of the spectral range, It can be concluded that the reflectance information acquired from the lower and upper bands of the spectrometer range were not useful for CBS identification.

The first derivatives of spectral reflectance for each class were shown (Fig. 4). Also the variation within the first derivatives for each class was demonstrated (Fig. 5). The spectral signatures of CBS-positive and CBSnegative classes created different slopes in the 490- to 590- and 630to $730-\mathrm{nm}$ bands, which are clearly highlighted (Fig. 4). The first derivative graphs for all four classes overlapped between 590 and $630 \mathrm{~nm}$, which suggested that their spectral reflectance at this band changed in a uniform manner. Between 637 and $670 \mathrm{~nm}$, the first derivatives for the healthy classes and the early stage of CBS had negative values; however, advanced stage of CBS generated positive first derivative values at the same band. The first derivatives of early stage of CBS and the two CBSnegative classes also overlapped and picked up to 0.6 within the wave band of 675 to $715 \mathrm{~nm}$; while the first derivatives of the advanced stage of CBS never reached over 0.2 at the same band. The first derivatives for advanced stage of CBS had the smallest standard deviations compared with other classes (Fig. 5) that suggested more within class spectral signature similarity for this class. At $690 \mathrm{~nm}$, the standard deviations of the first derivatives for the healthy classes as well as the early stage of CBS picked up to 0.35 while $t$ remained under 0.14 for the advanced stage of CBS at the same band.

COMPARISON BETWEEN REPETITIONS. The probability values 
for the two-sample $t$ test conducted for the spectral reflectance of every two consecutive repetitions and also between the first and last repetitions were illustrated (Table 1 ). The

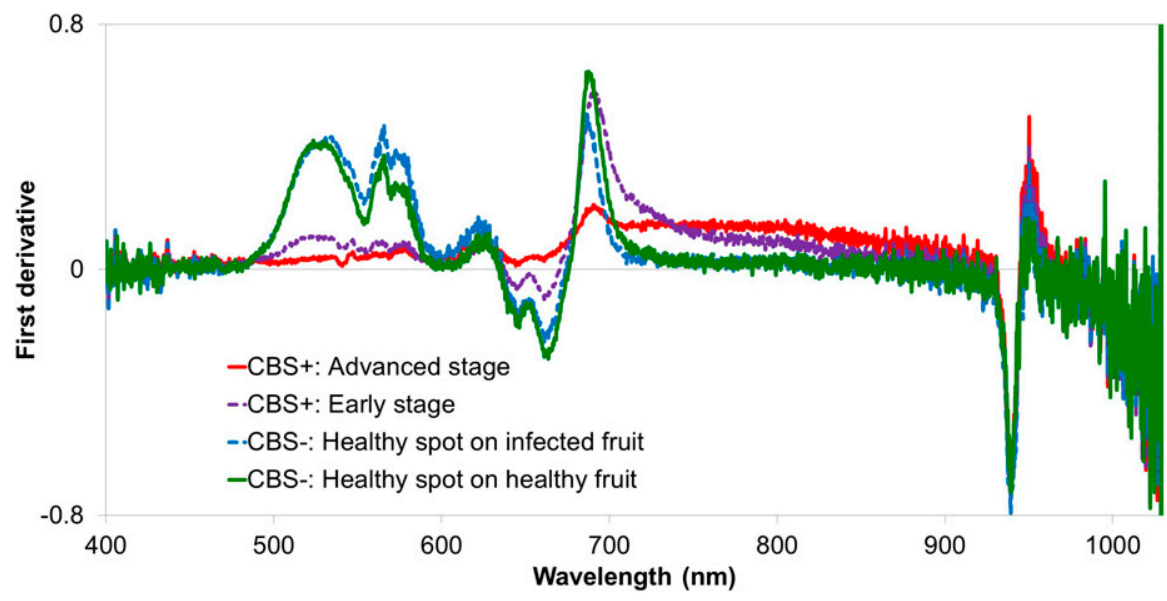

Fig. 4. Average first derivatives of spectral reflectance of samples in two citrus black spot positive (CBS-positive) classes (advanced and early stages of hard spot) and two CBS-negative classes (healthy spot on CBS-positive fruit and healthy spot on CBS-negative fruit) on peels of 'Valencia' sweet orange fruit within the visible and near-infrared spectral ranges.

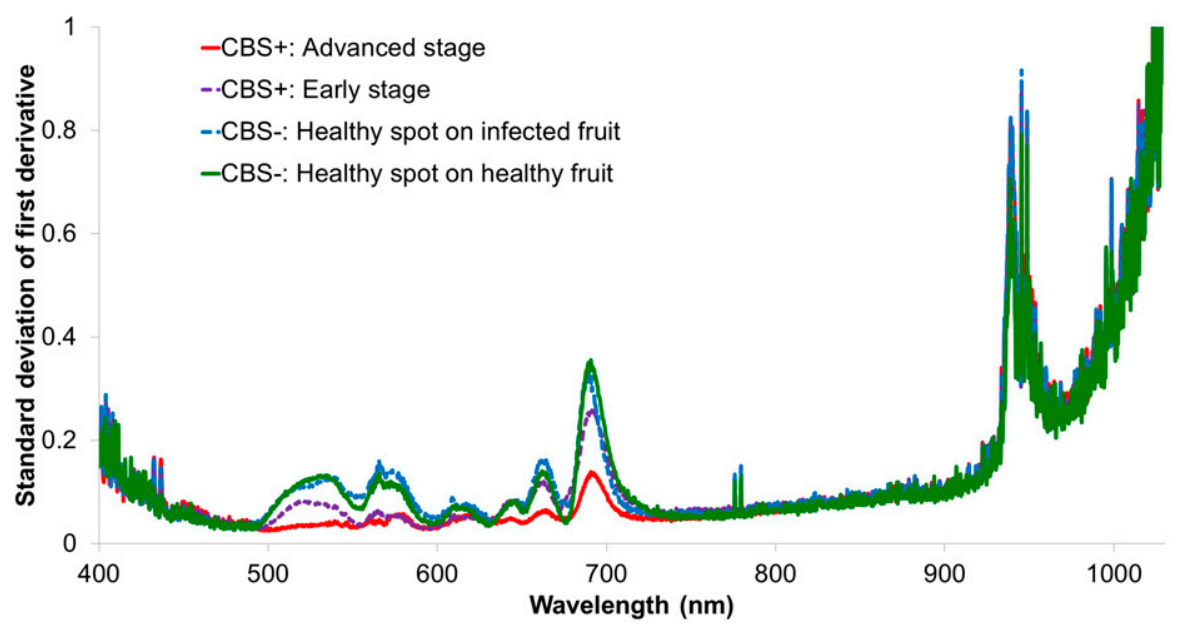

Fig. 5. Average standard deviations of first derivatives of spectral reflectance of samples in two citrus black spot positive (CBS-positive) classes (advanced and early stages of hard spot) and two CBS-negative classes (healthy spot on CBS-positive fruit and healthy spot on CBS-negative fruit) on peels of 'Valencia' sweet orange fruit within the visible and near-infrared spectral ranges. probability value less than 0.05 , it can be concluded that there was no significant difference between the repetitions.

The probability value for twosample $t$ test analyses conducted for the first derivatives of spectral reflectance of every two consecutive repetitions and also between the first and last repetitions were shown (Table 2 ). First derivative of a spectral reflectance describes the slopes in the spectral signature. No probability value below 0.05 was achieved for the two-sample $t$ tests. These results confirmed that the spectral signatures of samples in each did not significantly change over the period of 11 Apr. and 13 June 2014. It can be inferred that the spectral reflectance of CBS symptoms did not develop or disappear during the experiment period.

BAND SELECTION. The normalized coefficients of all wavelengths obtained by the feature ranking methods were illustrated (Fig. 6). Those wavelengths that had coefficients over 0.99 in all ranking methods were selected as the potential wavelengths. As shown in Fig. 6, wavelengths between 587 and $589 \mathrm{~nm}$ had coefficients over 0.99 in all methods. Therefore, this band was selected for the classification purpose.

The selected band (587 to 589 $\mathrm{nm}$ ) represents yellow color in the electromagnetic spectrum. As shown in Fig. 2, the spectral reflectances of CBS-positive and CBS-negative classes were distinctively separated between 587 and $589 \mathrm{~nm}$.

Classification. The classification results for all repetitions are shown in Table 3. The overall accuracies varied in a very narrow range from $93.3 \%$ to $94.6 \%$. It can be concluded that using the selected band as the input feature in the SVM classifier resulted in analogues

Table 1. Two-sample $t$ test probability values for pairwise comparison between the spectral reflectance of citrus black spot (CBS) lesions and healthy spots on the peels 'Valencia' sweet orange fruit for every pair of consecutive repetitions as well as the first and last repetitions of spectral measurements on 11 Apr., 18 Apr., 2 May, 15 May, 29 May, and 13 June 2014.

\begin{tabular}{lcccccc}
\hline & \multicolumn{5}{c}{ Pvalue for each pair of repetitions } \\
\cline { 2 - 6 } Classes & 1 vs. 2 & 2 vs. 3 & 3 vs. 4 & 4 vs. 5 & 5 vs. 6 & 1 vs. 6 \\
\hline Advanced stage CBS & 0.98 & 0.98 & 1.00 & 0.96 & 0.89 & 0.85 \\
Early stage CBS & 0.96 & 0.97 & 0.98 & 0.95 & 0.85 & 0.87 \\
Healthy spot on CBS sample & 0.99 & 1.00 & 0.98 & 0.95 & 0.96 & 0.87 \\
Healthy spot on healthy sample & - & 1.00 & 1.00 & 1.00 & 1.00 & 1.00 \\
\hline
\end{tabular}


Table 2. Two-sample $t$ test probability values for pairwise comparison between the first derivatives of the spectral reflectance of citrus black spot (CBS) lesions and healthy spots on the peels of 'Valencia' sweet orange fruit for every pair of consecutive repetitions as well as the first and last repetitions of spectral measurements on 11 Apr., 18 Apr., 2 May, 15 May, 29 May, and 13 June 2014.

\begin{tabular}{lcccccr}
\hline & \multicolumn{5}{c}{ Pvalue for each pair of repetitions } \\
\cline { 2 - 6 } Classes & 1 vs. 2 & 2 vs. 3 & 3 vs. 4 & 4 vs. 5 & $\mathbf{5}$ vs. 6 & 1 vs. 6 \\
\hline Advanced stage CBS & 0.84 & 0.84 & 1.00 & 0.59 & 0.39 & 0.76 \\
Early stage CBS & 0.91 & 0.82 & 0.82 & 0.70 & 0.27 & 0.40 \\
Healthy spot on CBS sample & 0.92 & 0.91 & 0.71 & 0.71 & 0.84 & 0.45 \\
Healthy spot on healthy sample & - & 1.00 & 1.00 & 1.00 & 1.00 & 1.00 \\
\hline
\end{tabular}

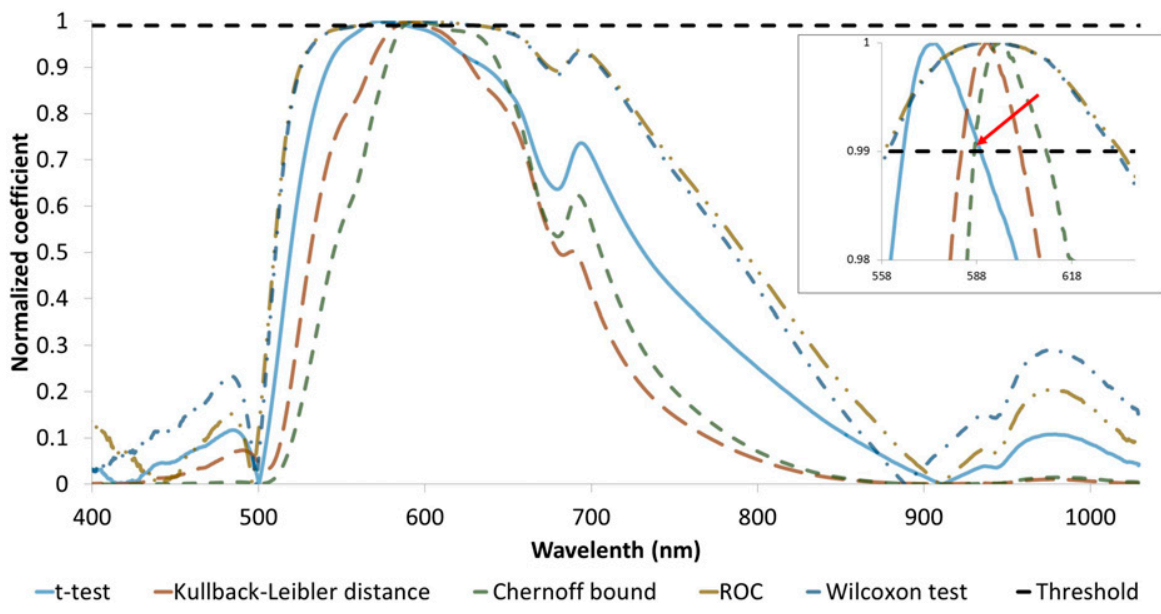

Fig. 6. Normalized coefficients of all wavelengths between 400 and $1030 \mathrm{~nm}$ that illustrated the level of relevance of each wavelength to classification of spots on the peels of 'Valencia' sweet orange fruit in the range of zero to one in which the wavelengths with normalized coefficient of zero had no contribution in the classification process, while the wavelengths with normalized coefficient of one had the maximum contribution in the classification process. The coefficients were acquired by five feature ranking methods [ $t$ test, Kullback-Leibler distance, Chernoff bound, receiver operating characteristic (ROC), and Wilcoxon test] and they were normalized by the maximum coefficient. The selected band is pointed by a red arrow.

classification accuracies in all repetitions. These results confirmed that there was no significant difference among the spectral reflectance of different repetitions. False negative rates varied from $6.9 \%$ to $9.2 \%$ for different repetitions while the false positive rates were between $0.5 \%$ and $4.6 \%$. The spectrometer probe has a very narrow core with the diameter of $600 \mu \mathrm{m}$. It is possible that the probe was pointed at the area around the hard spot, which was asymptomatic and as a result the spectral reflectance of an asymptomatic spot was measured instead of the hard spot. This measurement error might be one reason for higher false negative rates.

The results of a classification conducted for the entire dataset were shown (Table 4). An overall accuracy of $93.4 \%$ was achieved using the spectral data between 587 and $589 \mathrm{~nm}$. These results were similar to the classification accuracies achieved within each repetition. In most of cases, CBSnegative samples were identified with higher accuracies compared with CBSpositive samples. Again the false negative rate was equal to $9.5 \%$, which was higher than the false positive rate $(3.6 \%)$.

\section{Discussion}

Spectral reflectances of CBS lesions in two stages of the disease as well as two types of healthy spots were measured six times over 2 months in 2014. The results showed that the spectral signatures did not significantly change after the fruit were harvested. It can be derived that neither the stage of the disease changed after harvesting nor new lesion appeared on the healthy spots of CBS-infected fruit.

The wave band of 587 to $589 \mathrm{~nm}$ was selected as the most relevant band for detecting CBS. At this band, early and advanced stages of CBS reflected only $8.9 \%$ and $5.7 \%$ of light, respectively, while healthy spots reflected $30.5 \%$ of light on CBS infected and $26.7 \%$ of light on healthy fruit. Compared with healthy orange peel, black spots had darker color and as a result, they absorb more light. The standard deviation for CBS classes was $\approx 4.7 \%$ at the selected band, whereas it was $\approx 7.4 \%$ for healthy classes. This values confirmed more variation within each of healthy classes at the selected band. One reason might be the higher reflection from the healthy spots compared with the both CBS classes. All four classes had positive first derivatives ranging from 0.035 to 0.13 at the band of 587 to $589 \mathrm{~nm}$ that indicated an increasing trend for all spectral reflectance curves. The results also showed that the early and advanced stages of CBS at the band of 637 to $670 \mathrm{~nm}$ had opposite first derivative values. Therefore, the spectral information at this band might be useful for detecting the stage of CBS.

The spectral data at the selected band were used as the only input for classifying CBS and healthy spots in this study, which resulted in an acceptable overall accuracy of $93.4 \%$ while Bulanon et al. (2013) employed four wavelengths (three in visible band and one in NIR band) to achieve the accuracy of $96 \%$ in CBS detection. Although their results showed a higher accuracy, their proposed vision sensor required a multispectral camera, which will be more expensive and more difficult to use. The selected band in this research was within the visible band and a monochrome camera can be used to develop the vision sensor. Therefore, the final product might be 
Table 3. Number of spots on the peels of 'Valencia' sweet orange fruit that were classified into either citrus black spot positive $(\mathrm{CBS}+)$ or CBS-negative (CBS - ) classes along with their corresponding classification accuracies and misclassification error for six repetitions of the spectral measurements on 11 Apr., 18 Apr., 2 May, 15 May, 29 May, and 13 June 2014.

\begin{tabular}{|c|c|c|c|c|c|c|c|}
\hline \multicolumn{4}{|c|}{ First repetition (overall accuracy: $94.1 \%$ ) } & \multicolumn{4}{|c|}{ Second repetition (overall accuracy: $93.3 \%$ ) } \\
\hline \multicolumn{4}{|c|}{ Actual class [no. samples (\%)] } & \multicolumn{4}{|c|}{ Actual class [no. samples (\%)] } \\
\hline Prediction & CBS + & CBS - & Sum & Prediction & CBS + & CBS- & Sum \\
\hline CBS- & $27(9.2)$ & $184(99.5)$ & 211 & CBS- & $20(8.3)$ & $270(91.7)$ & 290 \\
\hline Sum & 292 & 185 & 477 & Sum & 278 & 278 & 556 \\
\hline
\end{tabular}

Third repetition (overall accuracy: $93.8 \%$ )

\begin{tabular}{|c|c|c|c|c|c|c|c|}
\hline \multirow{2}{*}{\multicolumn{4}{|c|}{ Actual class [no. samples (\%)] }} & \multirow{2}{*}{\multicolumn{4}{|c|}{ Actual class [no. samples (\%)] }} \\
\hline & & & & & & & \\
\hline Prediction & $\mathrm{CBS}+$ & CBS - & Sum & Prediction & $\mathrm{CBS}+$ & CBS- & Sum \\
\hline $\mathrm{CBS}_{+}$ & $263(91.3)$ & $10(3.6)$ & 273 & $\mathrm{CBS}_{+}$ & $258(93.1)$ & $10(3.8)$ & 268 \\
\hline Sum & 288 & 274 & 562 & Sum & 277 & 266 & 543 \\
\hline
\end{tabular}

Fifth repetition (overall accuracy: $93.9 \%$ )

\begin{tabular}{|c|c|c|c|c|c|c|c|}
\hline \multicolumn{4}{|c|}{ Actual class [no. samples (\%)] } & \multicolumn{4}{|c|}{ Actual class [no. samples (\%)] } \\
\hline Prediction & CBS+ & CBS - & Sum & Prediction & CBS + & CBS- & Sum \\
\hline $\mathrm{CBS}_{+}$ & $217(90.8)$ & $7(2.9)$ & 224 & $\mathrm{CBS}_{+}$ & $166(92.7)$ & $10(4.6)$ & 176 \\
\hline Sum & 239 & 240 & 479 & Sum & 179 & 217 & 396 \\
\hline
\end{tabular}

Table 4. Number of spots on the peels of 'Valencia' sweet orange fruit in the entire dataset of the citrus black spot (CBS) lesions and healthy spots (including all six repetitions of the spectral measurements on 11 Apr., 18 Apr., 2 May, 15 May, 29 May, and 13 June 2014), which were classified into either CBS-positive $(\mathrm{CBS}+)$ or CBS-negative $(\mathrm{CBS}-$ ) classes along with their corresponding classification accuracies and misclassification error.

\begin{tabular}{lccc}
\hline & \multicolumn{4}{c}{ All datasets (overall accuracy: 93.4\%) } \\
\hline \multicolumn{4}{c}{ Actual class [no. samples (\%)] } \\
\hline Prediction & CBS+ & CBS- & Sum \\
\hline CBS+ & $1,406(90.5)$ & $52(3.6)$ & 1,458 \\
CBS- & $147(9.5)$ & $1,408(96.4)$ & 1,555 \\
Sum & 1,553 & 1,460 & 3,013 \\
\hline
\end{tabular}

less expensive and more affordable for citrus growers.

\section{Conclusions}

In this study, the spectral characteristics of CBS symptoms were investigated and compared with the healthy tissue. A spectrometer was employed to measure the spectral reflectance of two CBS-positive and two CBS-negative samples in six repetitions over 2 months. The results showed that there was no significant difference among the spectral signatures of the same spot measured during 2 months of data collection. The wavelengths between 587 and 589 $\mathrm{nm}$ were identified as a potential wave band to develop a customized monochrome image acquisition system for in-field CBS diagnosis purpose. A
SVM classifier was trained with the spectral data from the selected wave band to classify the samples into two classes of CBS positive and CBS negative. The overall accuracies ranging from $93.3 \%$ to $94.6 \%$ were achieved for several repetitions of the experiment. Also, an overall accuracy of 93.4\% was achieved when all samples in the dataset regardless of their repetitions were used for the classification purpose. Bulanon et al. (2013) obtained an overall accuracy of $96 \%$ using the hyperspectral data in four bands, while in this study, only one band was used for classification with acceptable accuracy. A monochrome vision sensor may be developed based on the results of this study for in-field CBS diagnosis.

\section{Literature cited}

Bishop, C.M. 2006. Pattern recognition and machine learning. lst ed. Springer, New York, NY.

Bulanon, D.M., T.F. Burks, D.G. Kim, and M.A. Ritenour. 2013. Citrus black spot detection using hyperspectral image analysis. Agr. Eng. Intl. CIGR J. 15:171-180.

Dewdney, M.M., T.S. Schubert, M.R. Estes, and N.A. Peres. 2014. Florida citrus pest management guide: Citrus black spot. 10 Aug. 2015. <http://edis.ifas.ufl.edu/ CG088>.

Er, H., P. Roberts, J. Marois, and A. van Bruggen. 2013. Potential distribution of citrus black spot in the United States based on climatic conditions. Eur. J. Plant Pathol. 137:635-647.

Gottwald, T.R., J.V. da Graça, and R.B. Bassanezi. 2007. Citrus Huanglongbing: The pathogen and its impact. Plant Health Prog. doi:10.1094/PHP-20070906-01-RV.

Gottwald, T.R., J.H. Graham, and T.S. Schubert. 2002. Citrus canker: The pathogen and its impact. Plant Health Prog. doi:10.1094/PHP-2002-0812-01-RV.

Kotzé, J. 1981. Epidemiology and control of citrus black spot in South Africa. Plant Dis. 65:945-950.

Liu, H. and H. Motoda. 1998. Feature selection for knowledge discovery and data mining. 1st ed. Springer, Berlin, Germany. 


\section{Research Reports}

Pourreza, A., W.S. Lee, R. Ehsani, J.K. Schueller, and E. Raveh. 2015. An optimum method for real-time in-field detection of Huanglongbing disease using a vision sensor. Comput. Electron. Agr. 110:221-232.

Pourreza, A., W.S. Lee, E. Raveh, R. Ehsani, and E. Etxeberria. 2014. Citrus Huanglongbing detection using narrow-band imaging and polarized illumination. Trans. Amer. Soc. Agr. Biol. Eng. 57:259-272.

Qin, J., T.F. Burks, X. Zhao, N. Niphadkar, and M.A. Ritenour. 2012. Development of a two-band spectral imaging system for real-time citrus canker detection. J. Food Eng. 108:87-93.
Sankaran, S., A. Mishra, R. Ehsani, and C. Davis. 2010. A review of advanced techniques for detecting plant diseases. Comput. Electron. Agr. 72:1-13.

Schubert, T., M. Dewdney, N. Peres, M. Palm, A. Jeyaprakash, B. Sutton, S. Mondal, N.-Y. Wang, J. Rascoe, and D. Picton. 2013. First report of Guignardia citricarpa associated with citrus black spot on sweet orange (Citrus sinensis) in North America. Eur. J. Plant Pathol. 137:635-647.

Snedecor, G.W. and W.G. Cochran. 1989. Statistical methods. 8th ed. Iowa State Univ. Press, Ames, IA.
Stover, E., R. Driggers, M.L. Richardson, D.G. Hall, Y. Duan, and R.F. Lee. 2014. Incidence and severity of asiatic citrus canker on diverse citrus and citrus-related germplasm in a Florida field planting. HortScience 49:4-9.

Theodoridis, S. and K. Koutroumbas. 2009. Pattern recognition. 4th ed. Elsevier, Boston, MA.

Wang, X., G. Chen, F. Huang, J. Zhang, K.D. Hyde, and H. Li. 2012. Phyllosticta species associated with citrus diseases in China. Fungal Divers. 52:209-224. 